\title{
About quasi-solutions of traveling wave type in models for organizing cargo transportation ${ }^{1}$
}

\author{
Nerses K. Khachatryan \\ Senior Researcher, Laboratory of Dynamic Models of Economy and Optimization \\ Central Economics and Mathematics Institute, Russian Academy of Sciences; \\ Associate Professor, Department of Business Analytics \\ National Research University Higher School of Economics \\ Address: 47, Nakhimovsky Prospect, Moscow, 117418, Russian Federation \\ E-mail:nerses@cemi.rssi.ru; nkhachatryan@hse.ru

\section{Andranik S. Akopov} \\ Professor, Department of Business Analytics \\ National Research University Higher School of Economics; \\ Leading Researcher, Laboratory of Dynamic Models of Economy and Optimization \\ Central Economics and Mathematics Institute, Russian Academy of Sciences \\ Address: 20, Myasnitskaya Street, Moscow, 101000, Russian Federation \\ E-mail:aakopov@hse.ru
}

\section{Fedor A. Belousov}

Researcher, Laboratory of Dynamic Models of Economy and Optimization Central Economics and Mathematics Institute, Russian Academy of Sciences; Associate Professor, Department of Business Analytics

National Research University Higher School of Economics

Address: 47, Nakhimovsky Prospect, Moscow, 117418, Russian Federation

E-mail:sky_tt@list.ru; fbelousov@hse.ru

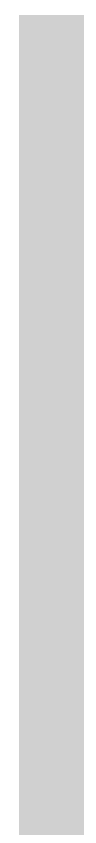

\begin{abstract}
This article is devoted to the construction and research of a model for organizing cargo transportation between two node stations connected by a railway line which contains a certain number of intermediate stations. The organization of freight traffic is facilitated by a number of technologies. These technologies determine the rules for taking on cargo at the initial node station, the rules of interaction between neighboring stations, as well as the rule of distribution of cargo to the final node stations. The process of cargo transportation is accompanied by the set rule of control consisting in measuring the volumes of goods transported at neighboring stations with a single time lag. For such a model, one must determine possible modes of cargo transportation and describe their properties. Traffic flow is described by a finite-difference analog of the nonlinear parabolic equation. The control system is set by nonlocal restrictions, which distinguishes the solutions of traveling wave type. The class of such solutions is extremely narrow. This results in the need for the "correct" extension of a class of solutions of the traveling wave type to a class of quasi-solutions of the traveling wave type. One type of expansion presupposes assumptions of discontinuous solutions of the traveling wave type; the second type allows for violations in a small control system. An essential lack of discontinuous solutions is their limitlessness. In this work, we investigate quasisolutions obtained with the help of a second type of extension. The distinctive feature of such quasisolutions is the assumption of feasibility of not local restrictions with the set error. The question of the limitation of such quasi-solutions is investigated. Using computer model implementation
\end{abstract}

\footnotetext{
${ }^{1}$ This work was partially supported by the Russian Foundation for Basic Research (project No. 16-01-00110)
} 
we investigate the dependence of the error in the performance of nonlocal restrictions on model parameters, which are the characteristics of the technologies used to carry out the cargo flow.

Key words: mathematical modeling of cargo transportation process, rule of control, differential equations, non-local restrictions, quasi-solutions, numerical realization.

Citation: Khachatryan N.K., Akopov A.S., Belousov F.A. (2018) About quasi-solutions of traveling wave type in models for organizing cargo transportation. Business Informatics, no. 1 (43), pp. 61-70.

DOI: 10.17323/1998-0663.2018.1.61.70.

\section{Introduction}

$\mathrm{O}$ ne of the key industries of any state is transport. One can distinguish among the main objectives of transport the following two: planning of transportation and organization of the process of transportation. For such an extensive territory as Russia, the second task becomes especially relevant. The set of research is devoted to the problem of organizing cargo transportation, which in particular, is considered in [1-7]. In [8-10] the model, an investigation is made of the organization of cargo transportation on the extended right of way with a large number of intermediate stations through which freight traffic passes. It is supposed that between two neighboring stations there is an interexchange railway track where part of cargo can temporarily be stored in special storage areas. We consider the capacity of such storage areas to be unlimited. The movement of cargo happens in one direction. The organization of freight traffic is carried out by means of two technologies. The first technology sets the rules for the interaction of neighboring stations, and the second - the rules for the interaction of stations with neighboring storage areas. One of the tasks of the second technology is to ensure uninterrupted cargo flow. A detailed description of these technologies is given in [8].

For cargo transportation, a simple control system is used. The amounts of processed freight for any planned interval of time at all stations should match a certain log of time, the same for all stations. Several variants of the model are considered. One of the variants of the model describes cargo transportation between two node stations and is given by the following system of differential equations with nonlocal linear restrictions [8]:

$$
\begin{gathered}
\dot{z}_{0}(t)=\psi_{1}(t)-\alpha z_{0}+\alpha z_{1}+\varphi_{0}\left(z_{0}\right), \quad t \in[0,+\infty) \\
\dot{z}_{i}(t)=\alpha z_{i-1}-2 \alpha z_{i}+\alpha z_{i+1}+\varphi\left(z_{i}\right) \\
i=1,2, \ldots, m, \quad t \in[0,+\infty) \\
\dot{z}_{m+1}(t)=\alpha z_{m}-\alpha z_{m+1}-\psi_{2}(t)+\varphi\left(z_{m+1}\right) \\
t \in[0,+\infty) \\
z_{i}(t)=z_{i+1}(t+\tau), i=0,1,2, \ldots, m, \quad t \in[0,+\infty)
\end{gathered}
$$

where $z_{i}(t)$ - the number of involved nodes at the $i$-th station at time $t, i=0,1, \ldots, m+1$.

Functions $\psi_{1}() \geq$.0 and $\psi_{2}() \geq$.0 determine, respectively, the intensity of handover of cargo on the initial node station and the intensity of the distribution of cargo from the final node station. Functions $\varphi_{0}($.$) and \varphi($.$) determine,$ respectively, speed of change of the number of involved processing nodes at the initial station and the number of involved processing nodes at other stations within the second technology. A description of the properties of the functions $\varphi_{0}($.$) and \varphi($.$) , and also their graphs is given in$ [8]. Here we note that these functions, as well as the functions $\psi_{1}($.$) and \psi_{2}($.$) are continuous.$

We will designate $c_{0}=-\dot{\varphi}_{0}(\Delta)$ and $c=-\dot{\varphi}(\Delta)$, where $\Delta$ is the number of involved processing nodes; when exceeded part of the cargo is sent to the storage area. Parameters $c_{0}$ and $c$ determine, respectively, the intensity of sending 
freight from the initial and intermediate stations to storage areas. Nonlocal linear restrictions (4) define a control system over the process of cargo transportation, and the parameter $\tau$ is its characteristic.

As noted in [8], the class of solutions of system (1) - (4) is extremely narrow. Therefore, its extension to the class of "quasi-solutions" was considered. The distinctive feature of the latter is the existence of gaps in a countable number of points. In [10] the existence and uniqueness theorem for quasi-solution is proved. The results of a numerical investigation of the system (1) - (4) are presented in [8]. The main task of the study was to study the dynamics of its quasi-solutions, as well as their dependence on some model parameters characterizing the control rule $(\tau)$, the technologies for transportation of cargo $(\alpha)$ and intensity of shipping cargo on a node station (function $\psi_{1}($.$) ). According to the$ results of numerical research, the quasi-solution systems (1) - (4) are majorized by exponential functions, whose growth decreases with increasing $\alpha$ and $\tau$. The parameter $\alpha$ values are limited due to the limited technological capabilities of the stations, and for large values $\tau$ the control system loses its relevance. Therefore, after a relatively short period of time, the number of involved nodes in the stations will become substantially larger than $\Delta$, which will entail failures in the organization of transportation. Thus, from a practical point of view, the limitlessness of quasi-solutions of system (1) - (4) is a lack of the model. We recall that quasi-solutions defined above, in contrast to solutions, contain gaps at points that are multiples of the characteristic of the control system. We call them quasisolutions of the first type.

Next, we consider another possible way of extending the class of solutions of the traveling wave type to a class of solutions of the "quasitravelling" wave type, namely, while weakening the nonlocal restrictions (4) (assuming that these restrictions are satisfied with some error).
Definition 1. The family of absolutely continuous functions $\left\{z_{i}(.)\right\}_{0}^{m+1}$, defined on $[0,+\infty)$, is called $\varepsilon$-quasi-solution of the traveling wave type of second type with characteristic $\tau>0$ for the system (1)-(4), if almost all $t \in[0,+\infty)$ functions $z_{i}($.$) satisfy the sys-$ tem of equations (1) - (3) and the condition $\left|z_{i}(t)-z_{i+1}(t+\tau)\right|<\varepsilon, i=0,1,2, \ldots, m, \quad t \in[0,+\infty) \quad$ is satisfied.

\section{On limited solutions of system (1) - (3)}

Before proceeding to the investigation of quasi-solutions of the system (1) - (4) of the second type, we study the set of all solutions of the system of equations (1) - (3). Obviously, for any initial conditions this system has a unique solution. Let us prove that for a certain choice of functions $\psi_{1}($.$) and \psi_{2}($.$) the solutions$ of the system of equations (1) - (3) are limited.

Theorem 1. Let the functions $\psi_{1}($.$) and \psi_{2}($.) be limited on a half-line $[0,+\infty)$. Then the solutions of the system of differential equations (1) - (3) are limited.

Proof. We will consider the first to be a component of the solution of a system of the differential equations (1) - (3), i.e. function $z_{0}($.$) . This function is either limited, or unlim-$ ited. We will assume that it is unlimited from the above. Then there is a sequence $\left\{t_{n}\right\} \rightarrow+\infty$ such that

$$
\lim _{n \rightarrow \infty} z_{0}\left(t_{n}\right)=+\infty \text { and } \lim _{n \rightarrow \infty} \dot{z}_{0}\left(t_{n}\right)>0 .
$$

Then it follows from equation (5), and in particular from the definition of the function $\varphi_{0}($.$) that$

$$
\lim _{n \rightarrow \infty} z_{1}\left(t_{n}\right)=+\infty \text { and } \lim _{n \rightarrow \infty} \dot{z}_{1}\left(t_{n}\right)>0,
$$

and also inequality

$$
\lim _{n \rightarrow \infty}\left[z_{1}\left(t_{n}\right)-z_{0}\left(t_{n}\right)\right]>0
$$

is carried out. 
From inequality (6) and the first equation of system of the differential equations (2) it also follows that

$$
\lim _{n \rightarrow \infty} z_{2}\left(t_{n}\right)=+\infty \text { and } \lim _{n \rightarrow \infty} \dot{z}_{2}\left(t_{n}\right)>0,
$$

and inequality

$$
\lim _{n \rightarrow \infty}\left[z_{2}\left(t_{n}\right)-z_{1}\left(t_{n}\right)\right]>0
$$

is carried out.

Acting by induction, we will find that

$$
\lim _{n \rightarrow \infty} z_{m+1}\left(t_{n}\right)=+\infty \text { and } \lim _{n \rightarrow \infty} \dot{z}_{m+1}\left(t_{n}\right)>0,
$$

and inequality

$$
\lim _{n \rightarrow \infty}\left[z_{m+1}\left(t_{n}\right)-z_{m}\left(t_{n}\right)\right]>0
$$

is carried out.

It follows from (7) - (8) that the left-hand side of equation (3) is positive, and the righthand side is negative. This contradiction is connected with the assumption of limitlessness of function $z_{0}($.$) from above. Thus, func-$ tion $z_{0}($.$) cannot be unlimited from the above.$ Similarly, it is possible to show that it cannot be unlimited from the below. So, function $z_{0}($.$) is$ limited.

Let us prove that the remaining components of the solution of the system of differential equations (1) - (3) will also be limited. We start with the function $z_{1}($.$) . We will assume that this$ function is unlimited from the above. Then there exists a sequence $\left\{t_{n}^{\prime}\right\} \rightarrow+\infty$ such that

$$
\lim _{n \rightarrow \infty} z_{1}\left(t_{n}^{\prime}\right)=+\infty \text { and } \lim _{n \rightarrow \infty} \dot{z}_{1}\left(t_{n}^{\prime}\right)>0 .
$$

Further, repeating the reasoning which is carried out at the proof of limitation of function $z_{0}($.$) , it is possible to show that take it$ takes the place of a ratio (7) and (8) where the sequence $\left\{t_{n}\right\}$ will be replaced with the sequence $\left\{t_{n}^{\prime}\right\}$. Therefore, for the chosen sequence $\left\{t_{n}^{\prime}\right\}$ the left part of the differential equation (3) will be positive, and right - negative. This contradiction is connected with the assumption of limit- lessness of function $z_{1}($.$) from the above. Thus,$ function $z_{1}($.$) also cannot be unlimited from$ the above. Similarly, it is possible to show that it cannot be unlimited from below. By an induction method, from the system of the differential equations (2) it is possible to show the limitation of functions $z_{i}(),. i=2,3, \ldots, m+1$.

So, according to the theorem 1 , at the limitation of functions $\psi_{1}($.$) and \psi_{2}($.$) , which is quite$ natural from the economic point of view, the solutions of the system of differential equations (1) - (3) are limited. For a more detailed investigation of the solutions, the system (1) - (3) was realized numerically with the help of the Runge-Kutta method of the fourth order. Before proceeding to a description of the numerical solution of this system, it is necessary to determine the functions $\varphi(),. \psi_{1}(t), \psi_{2}(t)$. Function $\varphi($.$) on a segment [0, \Delta]$ is given by a parabola $y=-a x^{2}+b x$, where $a>0, y(\Delta)=0$. Thus, the coefficients of the parabola are related by $b=a \Delta$. Obviously, the larger $a$, the greater the ordinate of the vertex of the indicated parabola, and, consequently, the intensity of reception of cargoes by the second technology. As functions $\psi_{1}\left(\right.$.) and $\psi_{2}($.) we will use two classes of functions:

a) constant function $\psi_{1}(t)=\psi_{2}(t)=d$;

b) periodic function $\psi_{1}(t)=\psi_{2}(t)=d+\gamma \cos (\omega t), d \geq \gamma$.

\section{Quasi-solutions of system (1) - (4) of the second type with constant functions $\psi_{1}($.$) and \psi_{2}($.}

We will consider the system of the differential equations (1) - (3) in which $\psi_{1}(t)=\psi_{2}(t)=d, d>0$. This means that on the initial node station freight traffic is carried out with constant intensity $d$ and with the same intensity as is distributed from the final node station. We will investigate the dependence of the solutions of the system (1) - (3) on the parameters of the model $\alpha, a, c_{0}, c, d$. Note that all these parameters are positive. According 
to results of numerical experiments, since at some time point, solutions of system (1) - (3) reach constant values for all values of parameters $\alpha, a, c_{0}, c, d$ this system, i.e. exists $\bar{t}>0$, it follows that conditions

$$
\begin{gathered}
z_{0}(t)=d_{0}, z_{1}(t)=d_{1}, \ldots, z_{m}(t)=d_{m}, \\
z_{m+1}(t)=d_{m+1}, t \in[\bar{t},+\infty),
\end{gathered}
$$

are satisfied, and numbers $d_{i}, i=0,1, \ldots, m, m+1$ satisfy the condition:

$$
d_{0}>d_{1}>\ldots>d_{m}>d_{m+1} .
$$

For example, one of solutions of system (1) - (3) is given in Figure 1.

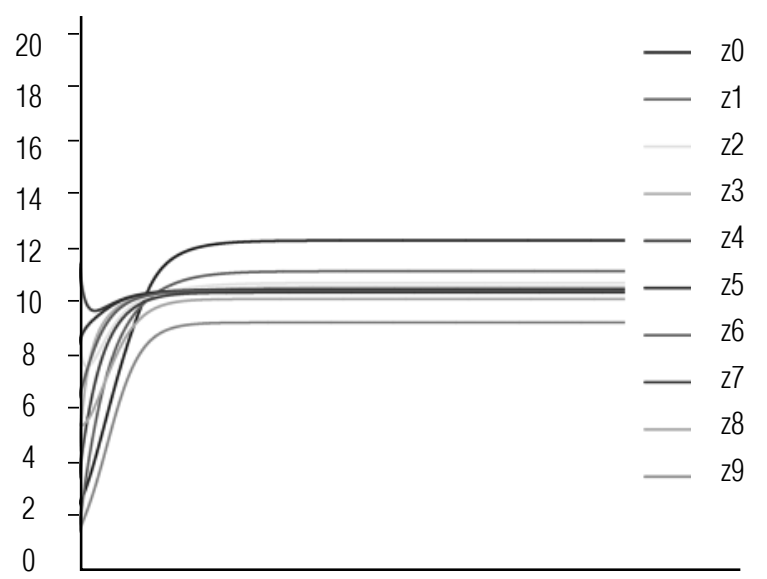

Fig. 1. The schedule of the solution of system (1) - (3) with constant functions $\psi_{1}($.$) and \psi_{2}($.

This solution is received at $\Delta=10$ and the following values of parameters: $a=0.2, c_{0}=c=1, d=3$.

We denote by $\bar{d}_{i}=d_{i}-\Delta, i=0,1, \ldots, m, m+1$. Thus, the numbers $\bar{d}_{i}$ determine the deviation of solutions from the value $\Delta$, that determines the capacity of the stations. In Figure 2 we give the histogram of the numbers $\bar{d}_{i}$ obtained from the solutions of the system (1) - (3), the graph for which is shown in Figure 1.

As can be seen from Figure 2, the first and last components of the solution of the system (1) - (3) have the greatest deviations from the

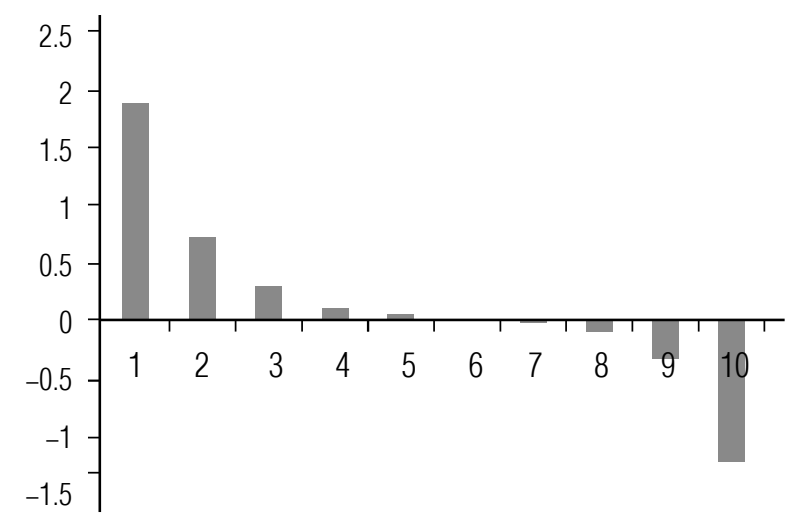

Fig. 2. The histogram of deviations of the solutions of system (1) - (3) from the value $\Delta$ (base case)

value $\Delta$. As we approach the central components of the solution, these deviations decrease, and both positive and negative deviations are present. We will give substantial interpretation of the histogram 2. For this purpose we will note that if the number of the involved nodes at the station is equal to $\Delta$, then it means that at this station the volume of infrastructure opportunities which allows us to organize uninterrupted freight traffic is completely used. A positive deviation from the value $\Delta$ indicates that the station connects additional capacities and at the same time there may be disruptions in the organization of cargo transportation, and negative - about the possibility of additional loading. Thus, the central stations make optimal use of infrastructure capabilities. The initial node station and the stations located near it are most loaded, and the final node station and the stations located near it do not fully utilize the infrastructure capabilities.

Let us investigate the dependence $\bar{d}_{i}$ on the parameters of the model. Let's start with the parameter $\alpha$. Recall that it defines the normative rules for the interaction of neighboring stations. We will increase the parameter $\alpha$ at constant values of other parameters. According to the results of experiments, the following regularity holds: the absolute value of the maximum deviation of the solution of system (1) - (3) 


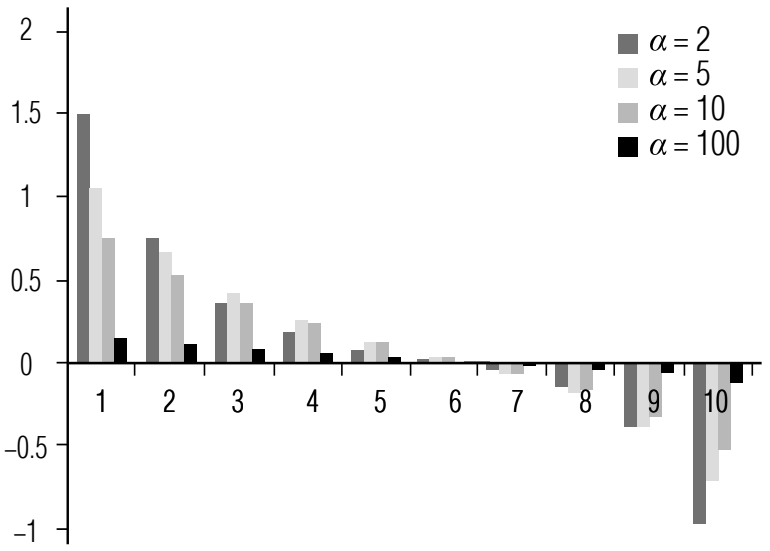

Fig. 3. The histogram of deviations of the solutions of the system (1) - (3) from the value $\Delta$ at change of parameter $\alpha$

from the value $\Delta$, i.e. $\max _{i=0,1, \ldots, m+1}\left|\bar{d}_{i}\right|$ decreases with increasing parameter $\alpha$, but for the central components of the solution these deviations can increase. This trend can be seen in Figure 3. It shows the histogram of deviations $\bar{d}_{i}$ for the parameter $\alpha$, equal to $2,5,10$ and 100 , and the following fixed values of the remaining parameters: $a=0.2, c_{0}=c=1, d=3$. Consequently, for arbitrarily small $\varepsilon>0$, there are values of the parameter $\bar{\alpha}$ such that for all $a \geq \bar{\alpha}$ (and for constant values of the other parameters) inequality

$$
\max _{i=0,1, \ldots, m, m+1}\left|\bar{d}_{i}\right| \leq \varepsilon
$$

will be carried out.

We will pass to a research of deviations $\bar{d}_{i}$ from the parameter $a$, which defines the intensity of receipt of goods by the second technology setting the rule of interaction of the station with neighboring storage areas. We will increase the value of this parameter at invariable values of other parameters. We recall that this leads to an increase in the intensity of receipt of goods on the second technology. As experiments show, this leads to a reduction of absolute values of negative deviations $\bar{d}_{i}$. At the same time, positive deviations $\bar{d}_{i}$ don't change. This tendency can be seen in Figure 4. It is the histogram of deviations $\bar{d}_{i}$ at the values of parameter $a$, equal to

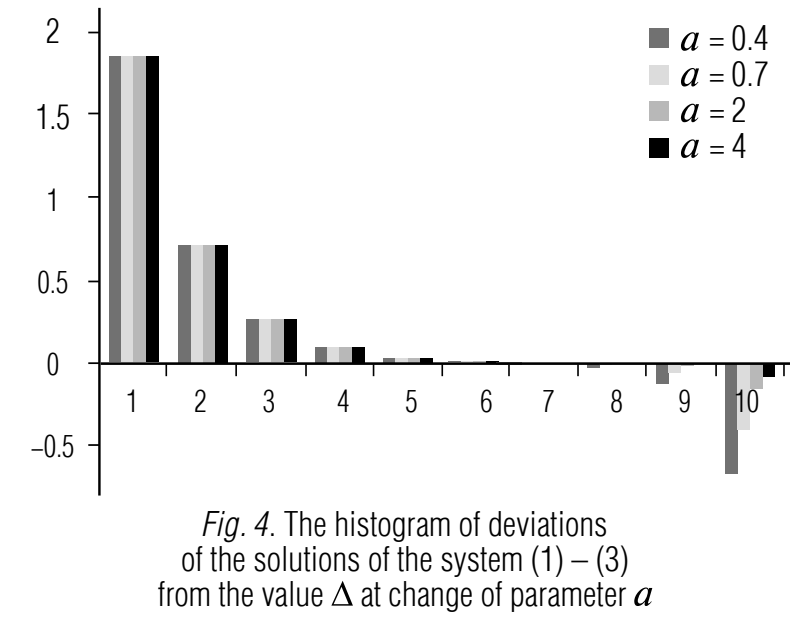

$0.4,0.7,2$ and 4 , and the following fixed values of other parameters: $\alpha=1, c_{0}=c=1, d=3$. We will note what, at such values of parameter $a$ and $\Delta=10$ the ordinate of vertex of a parabola accepts, respectively, the values 10 , 17.5, 50 and 100 .

We note that the parameter $a$ is present at all of the equation of system (1) - (3) except the first. Therefore, increasing parameter $a$, we increase the intensity of receipt of goods on the second technology at all stations, except for the initial one. It turns out that with the same effect, namely reduction of absolute values of negative deviations $\bar{d}_{i}$, it is possible to reach, changing parameter $a$ only in the last equation of system (1) - (3), i.e. increasing the intensity of receipt of goods on the second technology only at the final node station.

Let's proceed to a research of deviations $\bar{d}_{i}$ from the parameter $c_{0}$, that determines the intensity of departure cargoes from the initial node station to the storage area. We will increase the value of this parameter at constant values of other parameters. Recall that this leads to an increase in the intensity of departure goods from the initial node to the storage area. As experiments show, this leads to a decrease in positive deviations $\bar{d}_{i}$. In this case, negative deviations $\bar{d}_{i}$ do not change. This trend can be seen in Figure 5.

On the figure the histogram of deviations $\bar{d}_{i}$ at 


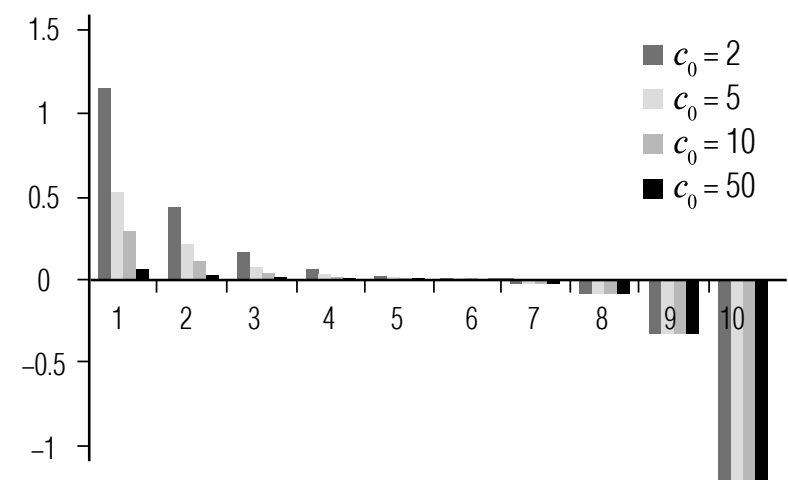

Fig. 5. The histogram of deviations of the solutions of the system (1) - (3) from the value $\Delta$ at change of parameter $c_{0}$

the values of parameter $c_{0}$, equal to $2,5,10$ and 50 and the following fixed values of the remaining parameters $\alpha=1, a=0.2, c=1, d=3$.

Thus, an increase in parameter $a$ in the last equation of system (1) - (3) and parameter $c_{0}$ leads to a reduction of an absolute value of the maximum deviation of the solution of system $(1)-(3) \max _{i=0,1, \ldots, m, m+1}\left|\bar{d}_{i}\right|$ from value $\Delta$. This in turn means, that for an arbitrarily small $\varepsilon>0$, there are values of the parameters $\bar{a}$ and $\bar{c}_{0}$, such that for all $a>\bar{a}, c_{0}>\bar{c}_{0}$ (and with constant values of the other parameters) inequality (9) will be satisfied.

Finally, we turn to the study of deviations $d_{i}$ from parameter $d$, representing the intensity of the delivery of cargo to the initial node station and the intensity of the distribution of cargo

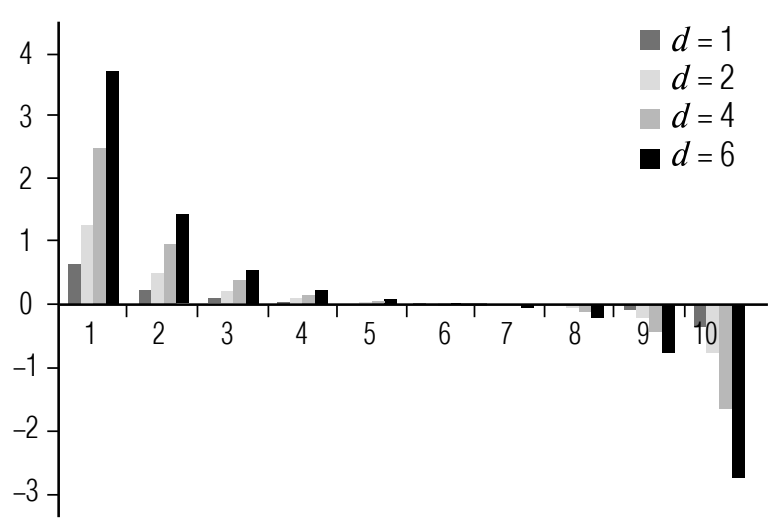

Fig. 6. The histogram of deviations of the solutions of the system (1) - (3) from the value $\Delta$ at change of parameter $d$ from the final node station. We will increase the value of this parameter at constant values of other parameters. As shown by experiments, this leads to an increase in the absolute values of the deviations $d_{i}$. This trend can be seen in Figure 6. On the figure, the histogram of deviations $\bar{d}_{i}$ at the values of parameter $d$, equal to 1 , 2, 4 and 6 and the following fixed values of the remaining parameters $\alpha=1, a=0.2, c_{0}=c=1$.

It should be noted that with decreasing parameter $d$ absolute values of deviations $d_{i}$ decrease to zero for $d=0$. In this case the solution of the system (1) - (3) converges to the stationary solution

$$
z_{0}(t)=z_{1}(t)=\ldots=z_{m}(t)=z_{m+1}(t) \equiv \Delta .
$$

This situation is shown in Figure 7.

We proceed to analyze the results obtained above with the help of numerical experiments. According to their results for as small as $\varepsilon>0$ by means of control parameters $\alpha, a, c_{0}$ it is possible to reach feasibility of inequality (9). A main objective of the conducted research is receiving $\varepsilon$-quasi-solutions of the traveling wave type with the characteristic $\tau>0$ for system (1) - (4), i.e. the organization of controlled freight traffic by means of the technologies defined above. It is easy to see that the solutions of system (1) - (3) satisfying condition (9) are $\varepsilon$-quasi-solutions of the traveling

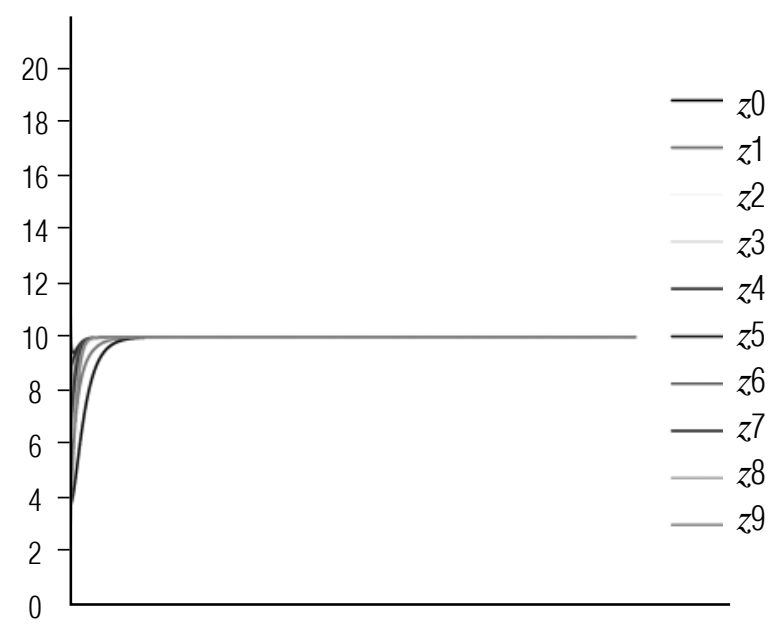

Fig. 7. The schedule of the solution of system (1) - (3) with $d=0$ 
wave type with an arbitrary characteristic $\tau>0$ for system (1) - (4).

Thus, we have revealed two methods of obtaining $\varepsilon$-quasi-solutions of the traveling wave type with an arbitrary characteristic $\tau>0$ for system (1) - (4). The first method is associated with the increase of the parameter $\alpha$, and the second with increasing parameters $a$ and $c_{0}$. Moreover, it is sufficient to increase the parameter $a$ only in the last equation of the system (1) - (3). Obviously, these two methods can also be combined with each other. From a practical point of view, the first method is associated with improving the infrastructure at all stations, and the second - with improving the infrastructure only at node stations, so that the latter is easier to use.

\section{Quasi-solutions of system (1) - (4) of the second type with periodic functions $\psi_{1}($.$) and \psi_{2}($.}

Let us consider a system of differential equations $(1)-(3)$, in which the functions $\psi_{1}($.$) and$ $\psi_{2}($.$) describing, respectively, the intensity of$ the supply of cargo to the initial node station and the intensity of the distribution of cargo from the final node station are periodic, i.e.

$$
\psi_{1}(t)=\psi_{2}(t)=d+\gamma \cos (\omega t), d \geq \gamma .
$$

According to the results of numerical experiments, starting from some time point $\bar{t}>0$, the solutions of the system (1) - (3) begin to oscillate in a certain neighborhood of the value $\Delta$, and the solution components satisfy the condition

$$
z_{0}(t)>z_{1}(t)>\ldots .>z_{m}(t)>z_{m+1}(t) \text { for any } t \in[\bar{t},+\infty) .
$$

Moreover, there exists a natural number $0<\bar{m}<m+1$ such that

$$
\begin{gathered}
z_{i}(t)>\Delta, \text { for } 0 \leq i \leq \bar{m}, t \in[\bar{t},+\infty), \\
z_{i}(t)<\Delta, \text { for } \bar{m}<i \leq m+1, t \in[\bar{t},+\infty) .
\end{gathered}
$$

For example, Figure 8 shows one of the solutions of system (1) - (3).

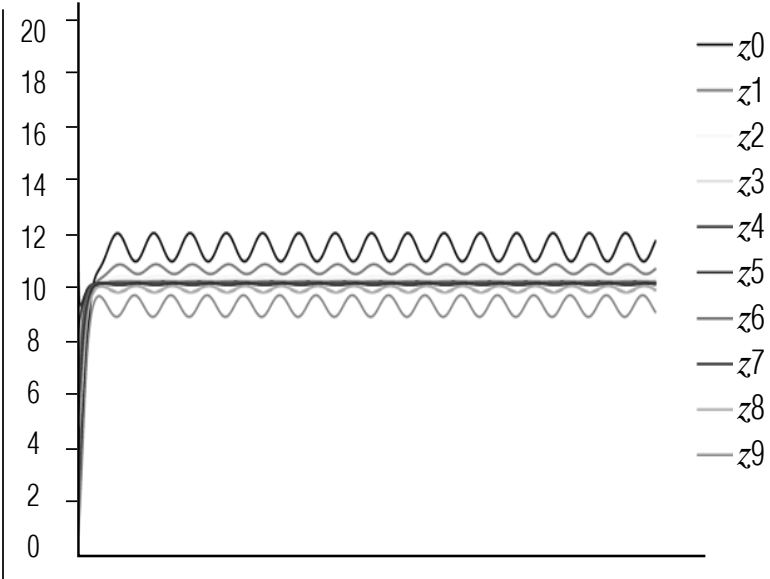

Fig. 8. The schedule of the solution of system (1) - (3) with periodic functions $\psi_{1}($.$) and \psi_{2}($.

This solution was obtained at $\Delta=10$ and the following values of the parameters

$$
\alpha=1, a=0.2, c_{0}=c=1, d=2, \gamma=1, \omega=1 .
$$

We denote by

$$
\bar{d}_{i}=\left\{\begin{array}{l}
\max _{t \in[\bar{t},+\infty)}\left(z_{i}(t)-\Delta\right), \text { if } 0 \leq i \leq \bar{m} \\
\min _{t \in[\bar{t},+\infty)}\left(z_{i}(t)-\Delta\right), \text { if } \quad \bar{m}<i \leq m+1 .
\end{array}\right.
$$

Thus, the number $\bar{d}_{i}$ determines the maximum deviation of the $i$-th component of the solution of the system (1) - (3) from the value $\Delta$, determining the capacity of the stations. It is obvious that the numbers $\bar{d}_{i}$ satisfy condition

$$
\begin{gathered}
\bar{d}_{0}>\bar{d}_{1}>\ldots>\bar{d}_{m}>\bar{d}_{m+1} ; \\
\bar{d}_{i}>0,0 \leq i \leq \bar{m} ; \bar{d}_{i}<0, \bar{m}<i \leq m+1 .
\end{gathered}
$$

Let us investigate the dependence $\bar{d}_{i}$ on the parameters of the model. Numerical experiments have shown that the dependence $\bar{d}_{i}$ on parameters $\alpha, a, c_{0}, c, d$ the same as in the case of constant functions $\psi_{1}($.$) and \psi_{2}($.$) . In par-$ ticular, by means of an appropriate choice of parameters $\alpha, a, c_{0}$ it is possible to make the absolute values of the deviations arbitrarily small. Thus, as in the case of constant functions $\psi_{1}($.$) and \psi_{2}($.$) , controlling these parame-$ ters, which determine the infrastructure capabilities of the stations, it is possible to organize a controlled freight flow. 


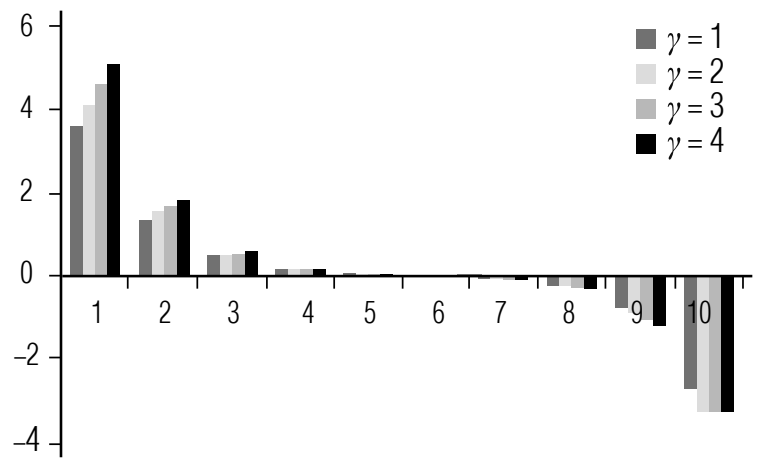

Fig. 9. The histogram of deviations of the solutions of the system (1) - (3) from the value $\Delta$ at change of parameter $\gamma$

It remains for us to investigate the dependence $\bar{d}_{i}$ on the parameters $\gamma$ and $\omega$. Unlike the parameters $\alpha, a, c_{0}$ only these parameters control does not allow us to make arbitrarily small absolute deviation values $\bar{d}_{i}$ and, accordingly, organize a controlled cargo flow. Nevertheless, we investigate the dependence $\bar{d}_{i}$ on $\gamma$ and $\omega$.

Let's start with the parameter $\gamma$. Note that this parameter determines the amplitude of the oscillations. We will increase the value of this parameter at constant values of other parameters. As experiments show, this leads to an increase in the absolute values of the deviations $\bar{d}_{i}$, i.e. dependence $\bar{d}_{i}$ from $\gamma$ is the same as on $d$. This trend can be seen in Figure 9.

The figure shows the histogram of deviations $\bar{d}_{i}$ at the values of parameter $\gamma$, equal to $1,2,3$ and 4 , and the following fixed values of other parameters $\alpha=1, a=0.2, c_{0}=c=1 \gamma=1$, $d=5, \omega=1$.
We will pass on to investigation of the dependence $\bar{d}_{i}$ on the parameter $\omega$. Since the period of functions $\psi_{1}($.$) and \psi_{2}($.$) is equal to$ $2 \pi / \omega$, then increasing the parameter $\omega$ leads to a decrease in the period of functions $\psi_{1}($.$) and$ $\psi_{2}($.$) . As shown by experiments, the depend-$ ence $\left|\bar{d}_{i}\right|$ on $\omega$ is a non-increasing function that takes values in a certain interval whose boundaries depend both on the index $i$, and on the remaining parameters of the model. Figure 10 shows a wide range of parameter variation $\omega-$ from 0.001 to 400 . The remaining parameters are fixed and take the following values: $\alpha=1, a=0.2, c_{0}=c=1 \gamma=1, d=2$. As can be seen from this figure, for example, the values $\bar{d}_{0}$ change in the interval lying in a segment $[1,2]$. It should be noted that for values $\omega$ close to zero functions $\psi_{1}($.$) and \psi_{2}($.$) are close$ to constants, therefore, the left boundary of the indicated intervals can be determined with the help of constant functions $\psi_{1}($.$) and \psi_{2}($.) (compare this histogram at $\omega=0.001$ with the histogram shown in Figure 2).

Finally, we analyze the results obtained in this section, i.e. in the case when the functions $\psi_{1}($.$) and \psi_{2}($.$) describing, respectively,$ the intensity of the supply of cargo to the initial node station and the intensity of the distribution of cargo from the final node station are periodic. As it turned out, these results do not qualitatively differ from the results obtained in the previous section, i.e. for the case when the functions $\psi_{1}($.$) and \psi_{2}($.$) are constants. For arbi-$

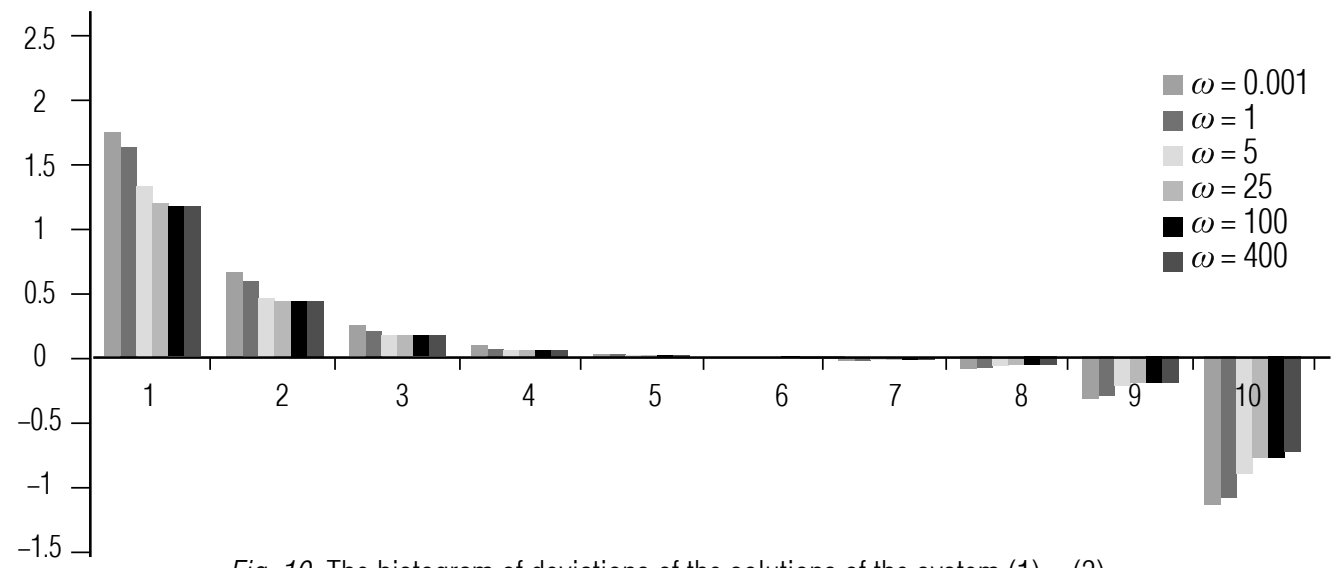

Fig. 10. The histogram of deviations of the solutions of the system (1) - (3) from the value $\Delta$ at change of parameter $\omega$ 
trarily small $\varepsilon>0$ we can obtain a $\varepsilon$-quasi-solution of the traveling wave type with the characteristic $\tau>0$ for system (1) - (3), i.e. organize a controlled flow of goods using the technologies described in the first paragraph. For this purpose, it is necessary to operate parameters $\alpha, a, c_{0}$, which is characteristic of the used technologies. The essence of this management comes down to improvement of the infrastructure of stations.

\section{Conclusion}

This article is devoted to the study of a model for organizing cargo transportation between two node stations carried out using a number of technologies with a set rule of control. Such a model is described by a finite-dimensional system of differential equations with non-local linear restrictions. The class of the solution satisfying nonlocal linear restrictions (solutions of the traveling wave type) is extremely narrow. This results in the need for the "correct" extension of class of solutions of the traveling wave type to a class of quasi-solutions of the traveling wave type. In [8-10] one of the methods of such an extension is given, thus making it possible to obtain quasi-solutions that differ from solutions by the presence of gaps in a countable number of points. The study of these quasisolutions has shown that they are unlimited. From a practical point of view, this is a serious flaw, since quasi-solutions describe the number of involved nodes in stations. In this connection, a different method for extending of class of solutions of the traveling wave type to a class of quasi-solutions of the traveling wave type is proposed. Quasi-solutions obtained with the help of this method differ from solutions in that they are allowed to satisfy nonlocal constraints with a given error. It is shown that such quasisolutions are limited.

Numerical experiments have shown that controlling the parameters of the model, one can obtain quasi-solutions for which nonlocal constraints are satisfied with arbitrarily small errors.

\section{References}

1. Galaburda V.G. (1983) Sovershenstvovanie tekhnologii perevozok i uvelichenie propusknoy sposobnosti zheleznykh dorog [Improvement of transportation techniques and increase in railroad traffic capacity]. Moscow: Moscow State University of Railway Engineering (in Russian).

2. Galaburda V.G. (1985) Optimal'noe planirovanie gruzopotokov [Optimal planning of cargo traffic]. Moscow: Transport (in Russian).

3. Kozovskii I.G. (1977) Ratsionalizatsiya perevozok gruzov na zheleznykh dorogakh [Improvement of railroad goods transportation]. Moscow: Transport (in Russian).

4. Levin D.Yu. (2010) Modelirovanie protsessov perevozki [Modeling of transportation processes]. World of Transport and Transportation, vol. 8, no. 5 (33), pp. 48-55 (in Russian).

5. Rubtsov A.O, Tarasov A.S. (2009) Modelirovanie zheleznodorozhnykh perevozok na territorii Rossii [Modeling of railway transport in the territory of Russia]. Proceedings of the Institute of System Analysis of Russian Academy of Sciences, no. 46, pp. 274-278 (in Russian).

6. de Jong G., Gunn H.F., Walker W. (2004) National and international freight transport models: an overview and ideas for further development. Transport Reviews, vol. 24, no. 1, pp. 103-124.

7. Yamada T., Russ B.F., Castro J., Taniguchi E. (2009) Designing multimodal freight transport networks: A heuristic approach and applications. Transportation Science, vol. 43, no. 2, pp. 129-143.

8. Khachatryan N.K., Akopov A.S. (2017) Model for organizing cargo transportation with an initial station of departure and a final station of cargo distribution. Business Informatics, no. 1 (39), pp. 25-35.

9. Beklaryan L.A., Khachatryan N.K. (2013) Ob odnom klasse dinamicheskikh modeley gruzoperevozok [On one class of dynamic transportation models]. Computational Mathematics and Mathematical Physics, vol. 53, no. 10, pp. 1649-1667 (in Russian).

10. Beklaryan L.A., Khachatryan N.K. (2006) Traveling wave type solutions in dynamic transport models. Functional Differential Equations, vol. 13, no. 2, pp. 125-155. 